\title{
Epitaxial multilayers of $\mathrm{YBa}_{2} \mathrm{Cu}_{3} \mathrm{O}_{x} / \mathrm{PrBa}_{2} \mathrm{Cu}_{3} \mathrm{O}_{x} / \mathrm{YBa}_{2} \mathrm{Cu}_{3} \mathrm{O}_{x}$
}

\author{
J. Gao, W.A.M. Aarnink, G.J. Gerritsma, A.J.H.M. Rijnders and H. Rogalla \\ Department of Applied Physics, University Twente, P.O. Box 217, 7500 AE Enschede. The Netherlands
}

\author{
F. Hakkens, W. Coene and M.A.M. Gijs \\ Philips Research Laboratories, 5600 JA Eindhoven. The Netherlands
}

Received 22 February 1991

Revised manuscript received 23 April 1991

\begin{abstract}
Heteroepitaxial multilayers of $\mathrm{YBa}_{2} \mathrm{Cu}_{3} \mathrm{O}_{x} / \mathrm{PrBa}_{2} \mathrm{Cu}_{3} \mathrm{O}_{x} / \mathrm{YBa}_{2} \mathrm{Cu}_{3} \mathrm{O}_{x}$ have been made by sputtering. No degradation of the transition temperature and the critical current density due to the presence of the $\mathrm{PrBa}_{2} \mathrm{Cu}_{3} \mathrm{O}_{x}$ layer could be observed. $\mathrm{By}$ using high-resolution transmission electron microscopy the atomic details of the interfaces and the defect structures have been studied. These films showed a perfectly stacked lattice just above the interface between film and substrate. The orientation of the $c$-axis perpendicular to the substrate was fairly perfect. The structural faults are mainly distributed in the middle and overlying layers. The dominant defects in our films seems to be stacking faults which give rise to nano-sized coherent anti-phase domains with the 1-2-3 structure. Rutherford backscattering spectroscopy, secondary ion mass spectroscopy, and scanning Auger microscopy were used to examine the interdiffusion between layers. Within the experimental resolution of $7 \mathrm{~nm}$ no interdiffusion is visible between $\mathrm{YBa}_{2} \mathrm{Cu}_{3} \mathrm{O}_{x}$ and $\mathrm{PrBa}_{2} \mathrm{Cu}_{3} \mathrm{O}_{x}$ layers.
\end{abstract}

\section{Introduction}

A basis for high- $T_{\mathrm{c}}$ superconducting Josephson devices can be formed by the growth of multilayer structures of superconductors and nonsuperconductors. In general, the different materials should be able to withstand high-temperature processes without pronounced interdiffusion and be chemically compatible with one another. The surface morphology of the multilayer is of primary concern. Of the electrical properties both high transition temperature and high critical current density are essential. Since $\mathrm{PrBa}_{2} \mathrm{Cu}_{3} \mathrm{O}_{x}$ (PBCO) has the same perovskite structure and similar lattice constant as $\mathrm{YBa}_{2} \mathrm{Cu}_{3} \mathrm{O}_{x}$ (YBCO), it can be easily grown epitaxially on the underlying $\mathrm{YBCO}$ and enables heteroepitaxial growth of the overlying YBCO layer [1]. So the YBCO/ $\mathrm{PBCO} / \mathrm{YBCO}$ sandwich structure is of great interest for SNS device applications.

We have prepared YBCO/PBCO/YBCO trilayers in situ by modified off-axis sputtering. Based on these epitaxially grown multilayers, all-high- $T_{\mathrm{c}}$ SNS-type Josephson junctions and DC SQUIDs have been successfully fabricated $[2,3]$. The composition of interfaces plays a very important role and strongly affects the performance of high- $T_{\mathrm{c}}$ superconducting devices. So the research on the microstructure and interfaces of the YBCO/PBCO/YBCO multilayer represents an important task. In this paper we report on a detailed study of the microstructure and the interfaces of these heterostructures. High-resolution transmission electron microscopy (HRTEM), Rutherford backscattering spectroscopy (RBS), He-ion channeling, scanning Auger microscopy (SAM), and secondary ion mass spectroscopy (SIMS) were used as analysis techniques.

\section{Experimental}

Both YBCO and PBCO thin layers are deposited in situ by a modified off-axis RF-magnetron sputtering technique. Details of this technique have been described in a previous publication [4]. Stoichiometric sintered YBCO and PBCO targets with a diameter of $50 \mathrm{~mm}$ have been used. The substrates we 
used in this study are $\mathrm{Y}$-stabilized $\mathrm{ZrO}_{2}(100)$. The sputter gas is a mixture of argon and oxygen which consists, in most cases, of 50\% oxygen and $50 \%$ argon. Typical sputter pressures are between $5 \times 10^{-2}$ and $3 \times 10^{-1}$ mbar. During deposition the substrates were heated to $640-700^{\circ} \mathrm{C}$. The incident RF sputter power density was between 1 and $5 \mathrm{~W} / \mathrm{cm}^{2}$. This procedure consistently produces $\mathrm{YBCO}$ thin films showing zero resistance $T_{\mathrm{c}, z e r o}$ around $90 \mathrm{~K}$ and $J_{\mathrm{c}}$ $\left(77 \mathrm{~K}\right.$ ) above $10^{6} \mathrm{~A} / \mathrm{cm}^{2}$. Layers of PBCO have been prepared using the same deposition procedures. To avoid contamination of the interfaces of the sandwich structures, we used a multi-target arrangement in our sputter system; so the substrates could be turned under different targets and various materials can be deposited sequentially without breaking the vacuum. After film deposition an anneal at $400^{\circ} \mathrm{C}$ in one bar oxygen was carried out.

For measuring resistivity and critical current the films have been photo-lithographically structured into small bridges of $100 \mu \mathrm{m}$ length and $10 \mu \mathrm{m}$ width by wet chemical etching in diluted phosphoric acid. The structuring process caused less than $1 \mathrm{~K}$ reduction of $T_{\mathrm{c}}$. As a criterion for the determination of the critical current, a resistance of $1 \times 10^{-4}$ of the normal state resistance at $100 \mathrm{~K}$ was used.

To study the phase formation and microstructure. in these multilayers, X-ray diffraction, RBS, He-ion channeling, and cross sectional HRTEM have been used. The interdiffusion between YBCO and PBCO layers was investigated by SAM, RBS, and SIMS. Analysis of the RBS spectra was done by the RUMP computer code [5], implemented on a $80386 / 80387$ PC. The results of SAM were analysed using the ESAU program implemented on a PDP 11 computer controlling the Phi Multiprobe 600 systems [6]. The base pressure of this system is $3 \times 10^{-6}$ mbar. The Auger spectra, depth profiles and line profiles were taken using a $10 \mathrm{keV}$ electron beam with a typical beam current of $0.5 \mu \mathrm{A}$. For the ion etching $3.5 \mathrm{keV}$ $\mathrm{Ar}^{+}$ions were used. During calibration the $\mathrm{Ar}^{+}$ion gun is aligned with the electron beam, so that depth profiles are taken at the center of the sputter crater (see the inset schematic diagram in fig. 8). The sputter rate has been calibrated using a $\mathrm{Ta}_{2} \mathrm{O}_{5}$ layer with a thickness of $1000 \AA$, grown on a Ta substrate. Also the results of RBS measurements have been used to determine the sputter rate of the $\mathrm{Ar}^{+}$ion gun. For crater edge profiling, line profiles were recorded at the sputter crater edge along curve $L$ (fig. 8). Calculations show (see below) that the angle between the normal to the sample surface and the normal to the surface of the crater edge equals $\sim 0.04^{\circ}$. Therefore, interfaces are stretched at the crater edge and can be studied in greater detail. Moreover, scanning electron microscopy (SEM) and $\alpha$-step profiler were used to study the surface quality.

\section{Results and discussions}

In a previous paper we have shown that the transition temperature of the YBCO layers is not degraded by the presence of the PBCO middle layer. The transition temperature $T_{\text {c.zero }}$ of both YBCO overlying and underlying layers was found to be above $90 \mathrm{~K}$ as measured on $\mathrm{YBCO} / \mathrm{PBCO} / \mathrm{YBCO}$ trilayers structure with an Ar-ion etching technique [7]. Here we further present the results of the critical current density measurements, which are indicative for the growth quality of superconducting layers. The usual four-probe method was used. The thicknesses of YBCO layers in the multilayer structure were estimated from deposition times. The temperature dependence of the critical current density of a YBCO thin film with a thickness of $50 \mathrm{~nm}$, covered by a $30 \mathrm{~nm}$ thick epitaxial PBCO layer, is shown in fig. 1. At the boiling temperature of liquid nitrogen the critical current density was $1.5 \times 10^{6} \mathrm{~A} / \mathrm{cm}^{2}$. This result is comparable to results on our YBCO single layer with the same film thickness. Therefore it can be concluded that the good superconducting properties of YBCO layers are still preserved throughout the whole fabrication procedure.

For the individual YBCO layer, a metallic behavior is indicated by the residual resistivity ratios (RRR) ranging between 2.5 and 3 and by resistivity values at $100 \mathrm{~K}$ of $35-100 \mu \Omega \mathrm{cm}$. The normal-state resistivity of the multilayer structure is significantly higher. However, when the YBCO overlying layer and $\mathrm{PBCO}$ middle layer are etched away by an $\mathrm{Ar}$ ion beam, the resistivity at $100 \mathrm{~K}$ of the YBCO underlying layer is found to be similar to that of the individual YBCO thin films. The RRR value of the multilayers is slightly decreased whereas the transitions are still as sharp as those of single YBCO thin 


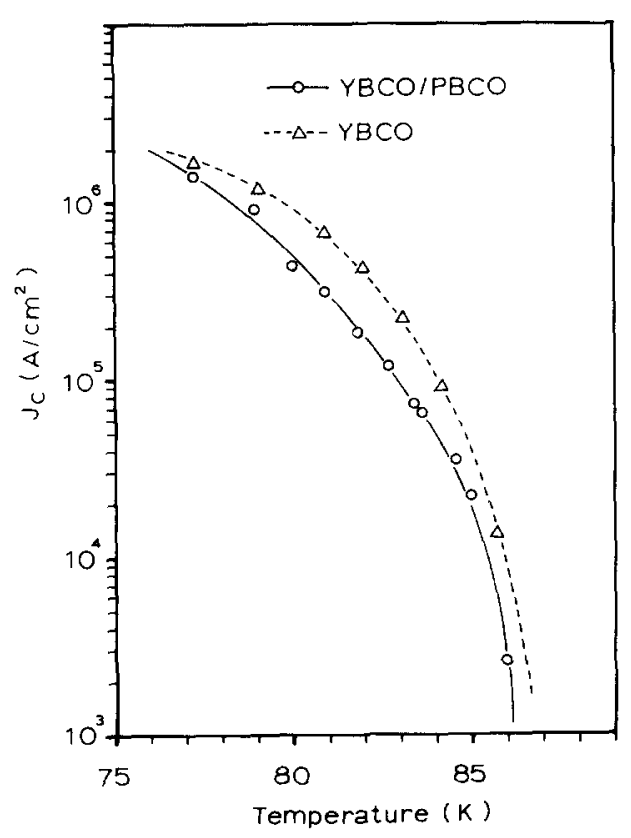

Fig. 1. The temperature dependence of the critical current density. The solid line is the curve of a $50 \mathrm{~nm}$ thin YBCO film covered by $30 \mathrm{~nm}$ thick epitaxial layer of PBCO. The dotted line is the data of $50 \mathrm{~nm}$ thin YBCO film without PBCO coverlayer.

films. We attributed this decrease to the relative higher defect density in the PBCO and YBCO overlying layers.

$\mathrm{X}$-ray diffractograms always reveal high orientation with the $c$-axis perpendicular to the substrate, as in the case of individual YBCO and PBCO layers. In fig. 2 we compared the $X$-ray diffraction pattern of a complete $\mathrm{YBCO} / \mathrm{PBCO} / \mathrm{YBCO}$ trilayer grown on $\mathrm{ZrO}_{2}$ substrate to that of $\mathrm{YBCO}$ and $\mathrm{PBCO}$ single layers. Typical substrate and [00/] peaks of $1-2-3$ compound are seen. On the diffraction pattern of PBCO thin film a few extra peaks are observed (as marked by asterisks). One belongs to the [ 110$]$ or [103] peaks of 1-2-3 structure which sometimes appear also in the X-ray pattern of YBCO films. Another peak seems to be a foreign phase which was formed during growth of the 1-2-3 structure of PBCO. However, we noticed that these extra peaks disappeared on the spectra of multilayers. The reason is that the PBCO layer in the multilayer is much thinner than this PBCO thin film (the thickness of the former is $\sim 30 \mathrm{~nm}$, the latter is $\sim 150 \mathrm{~nm}$ ). Therefore the content of coherent precipitates of any

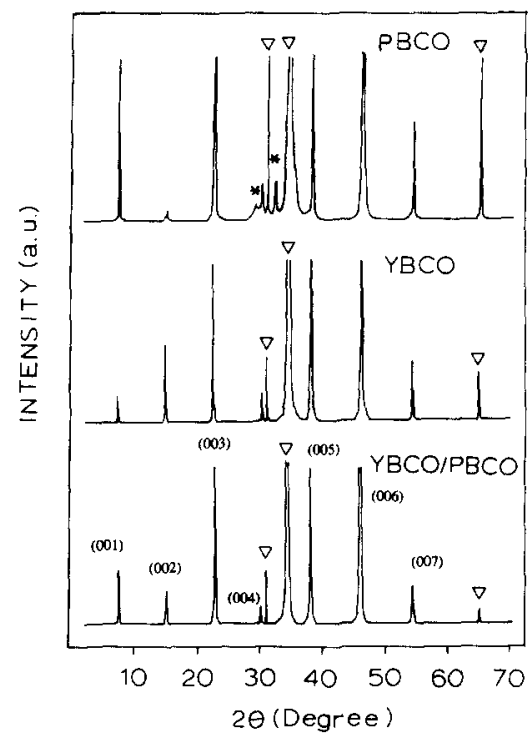

Fig. 2. X-ray diffraction patterns of $\mathrm{YBCO}, \mathrm{PBCO}$ individual layers, and $\mathrm{YBCO} / \mathrm{PBCO} / \mathrm{YBCO}$ trilayer grown on (100) $\mathrm{ZrO}_{2}$ substrates. The peaks originating from the substrate are marked by down triangles and the extra peaks are marked by asterisks.

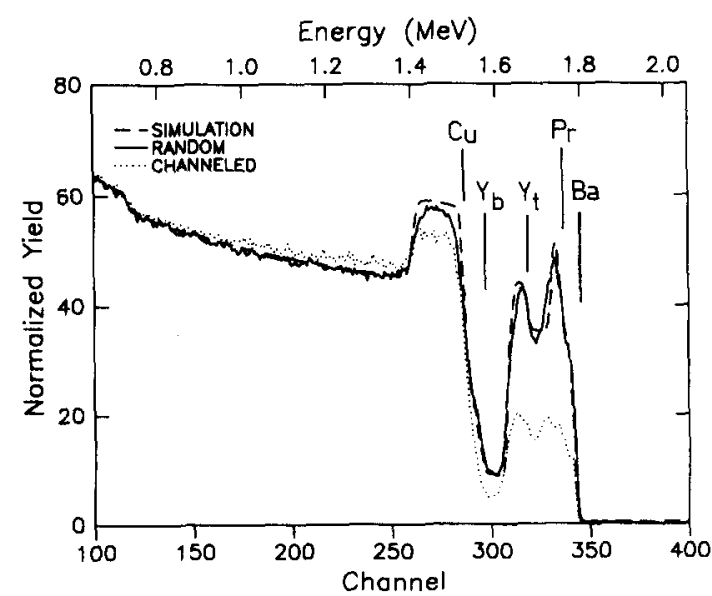

Fig. 3. The RBS and $\mathrm{He}^{+}$ion channeling spectroscopy of a $\mathrm{YBCO} /$ $\mathrm{PBCO} / \mathrm{YBCO}$ trilayer sample with thickness of 114,34 and 40 $\mathrm{nm}$ from substrate to top, respectively. The computer simulation curve fitting well to the experimental data indicates good overall stoichiometry. Within the depth resolution limits of RBS of 5 $\mathrm{nm}$, no interdiffusion between layers could be observed.

kind is negligibly low in the multilayers, otherwise they could be detectable in X-ray spectra.

The channeling analysis using $2.0 \mathrm{MeV}$ He-ions 
aligned parallel to the [001] direction of the film demonstrate that epitaxy is maintained throughout the three layers. A minimum yield value $\chi_{\min } \sim 35 \%$, defined as the ratio of the backscattering yields for perfect alignment with the $\langle 001\rangle$ crystal direction to that for random incidence throughout all three layers and substrate, has been measured. This value is in agreement with the result on $\mathrm{YBCO} / \mathrm{PBCO} /$ YBCO trilayers presented by Schubert et al. [8] but is considerably higher than the value of YBCO thin films. The relative higher minimum yield value $\chi_{\min }$ is attributed to the presence of the PBCO middle layer. At the interfaces the difference in lattice constants between YBCO and PBCO, even though it is very small, would introduce the formation of misfit dislocations. So most of the contribution to interface dechanneling might be due to scattering by disordered misfit dislocations.

The microstructure of these multilayers has been studied by TEM with an atomic resolution using a Philips CM30/Super Twin microscope. In fig. 4 a cross-sectional TEM bright-field image of the com-

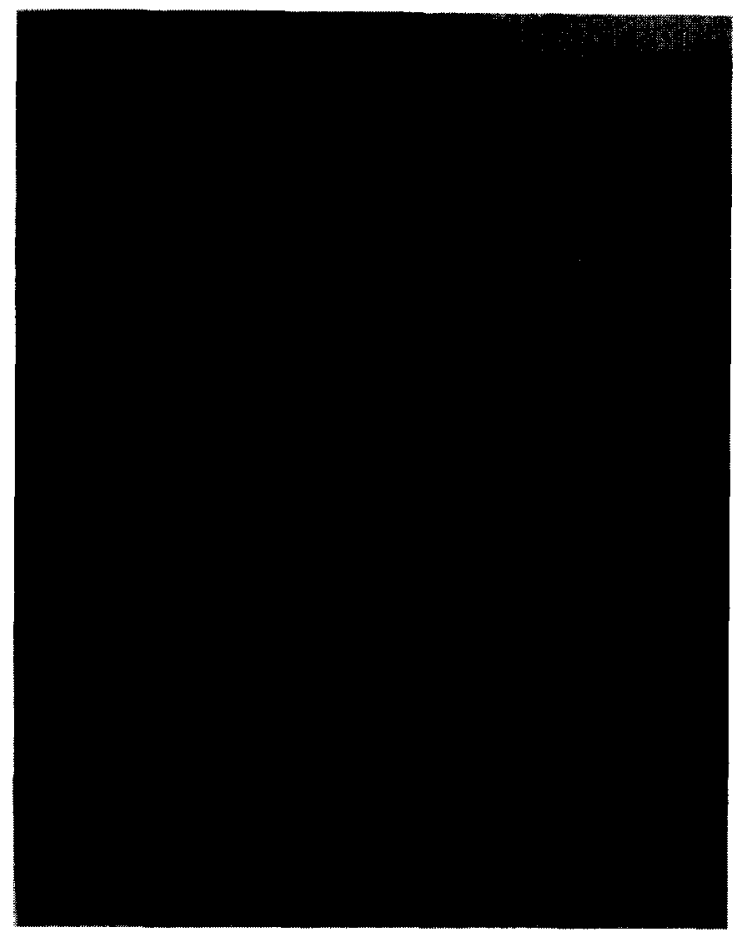

Fig. 4. Cross-sectional TEM bright-field image of the complete YBCO/PBCO/YBCO trilayer plete deposited $\mathrm{YBCO} / \mathrm{PBCO} / \mathrm{YBCO}$ trilayer on a (100) $\mathrm{ZrO}_{2}$ substrate is shown. From RBS measurements we determined the layer thicknesses in this sample to be $107 \mathrm{~nm}$ YBCO, $53 \mathrm{~nm}$ PBCO, and 40 $\mathrm{nm}$ YBCO, respectively, from substrate to top. The measured film thicknesses are in rather good agreement with that shown in the TEM photograph. Stacking defects are seen in this bright-field image as horizontal fringes. Close to the substrate, only few defects are observed. Further away from the substrate/film interface, the defect density increases. The $\mathrm{YBCO} / \mathrm{PBCO}$ interfaces are not visible in fig. 4, since their contrast is largely obscured due to the higher defect density in that area.

The HRTEM image in fig. 5 reveals the atomic stacking at the interface region between the substrate and the first YBCO layer of the film. The image is made with the electron beam parallel to the [0 10$]$ orientation of YBCO, as is clear from the inset. The focus condition of the microscope is chosen such that $\mathrm{Y}, \mathrm{Ba}$ and $\mathrm{Cu}$ atoms are properly imaged as white dots. The stacking sequence of $\mathrm{YBCO}$ is indicated. In the layer just above the substrate, a perfect and defect free YBCO stacking with $c$-axis texture is revealed in HRTEM, in agreement with the bright-field observations. Looking in more detail at the interface area in fig. 5 , it is found that no real coherent match is realized between film and substrate, and that the interface is not perfectly sharp at an atomic scale. Interface steps are present as can be observed in the bottom-right area of fig. 5. Also local small orientation differences are revealed in the substrate area, which have surprisingly no effect on the YBCO epitaxy. This can be seen in the bottom-left area of fig. 5. The substrate diffraction spots, which are marked by " $S$ " in the inset of fig. 5 , are also slightly misaligned with respect to the YBCO spot. Indeed, only the alignment in the [001] direction of the film normal is clearly maintained. Therefore, it seems that the film quality is not critically dependent on the substrate conditions. Effects due to possible reactions between film and substrate are improbable. Similar observations of the robustness of the YBCO epitaxy with respect to the substrate have been made in the case of thin films grown on $\mathrm{MgO}$ as reported by Streiffer et al. [9].

The defect-free layer of the first grown film is estimated around $30 \mathrm{~nm}$. This corresponds to the 


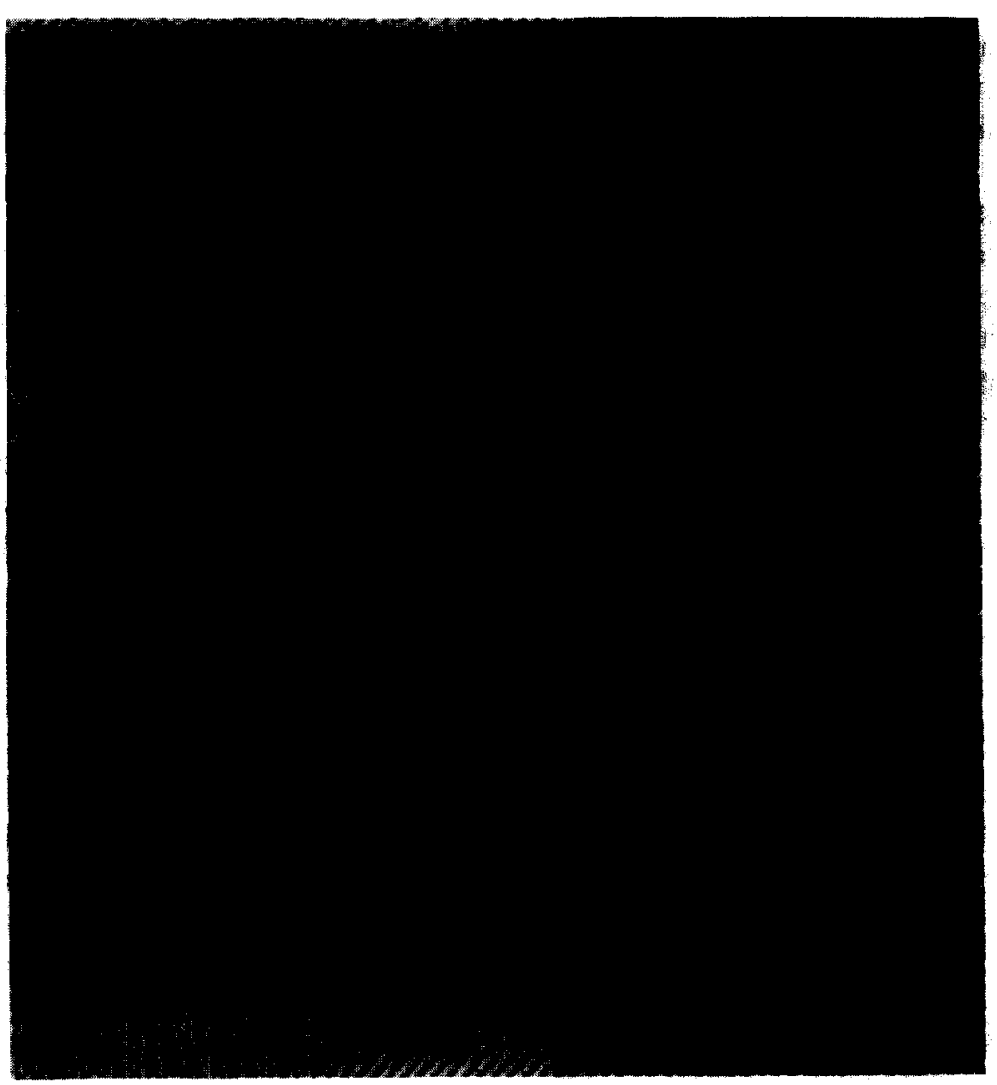

Fig. 5. HRTEM image of the interface of the substrate and the first YBCO layer. The corresponding electron diffraction pattern characterizes the epitaxy of the film. The $c$-axis adopts the orientation along the $\langle 100\rangle$-direction of the substrate.

thickness range of the YBCO thin films, which are usually made by our preparation technique $[4,7]$ : it illustrates the excellent quality of our ultra-thin YBCO films.

Figure 6 shows a HRTEM image in the $[010]$ orientation in the defect region of the film, further away from the substrate. The focus condition is different from that of fig. 5 , and the position of the atom planes is indicated by the arrows. Looking under grazing incidence along the arrows clearly reveals the local stacking defects, which are present in small domains with a size of $5-10 \mathrm{~nm}$. The single rows of white dots in fig. 6 for the undistorted YBCO lattice, represent the $\mathrm{Cu}-\mathrm{O}$ planes sandwiched between two Ba planes. In the defect domains, the single rows are locally continued by double rows of white dots. At first intuition, these defects are reminiscent of those related to the well-known 1-2-4 defects [10], in which an extra $\mathrm{Cu}-\mathrm{O}$ plane is inserted between the $\mathrm{Ba}-\mathrm{O}$ planes in the 1-2-3 structure. However, close inspection shows that the repeat distance along [001] in the defect area remains identical to $c$ of YBCO. Therefore, these defect areas are identified as small anti-phase domains with a displacement along [001] of around $0.97 \mathrm{~nm}$ with respect to the undistorted YBCO lattice. At the boundaries of these small domains, the surrounding (001) planes in the 1-2-3 lattice are bent, giving rise to dislocation-type contrast. The scale of the defect domains is of the same order of magnitude as the superconducting coherence lengths of YBCO material (around $2 \mathrm{~nm}$ ). Therefore, they could act as flux pinning centers for magnetic flux lines, hereby enhancing the critical current density [11-13].

Another sample which was prepared with lower deposition temperature and higher RF power seems 


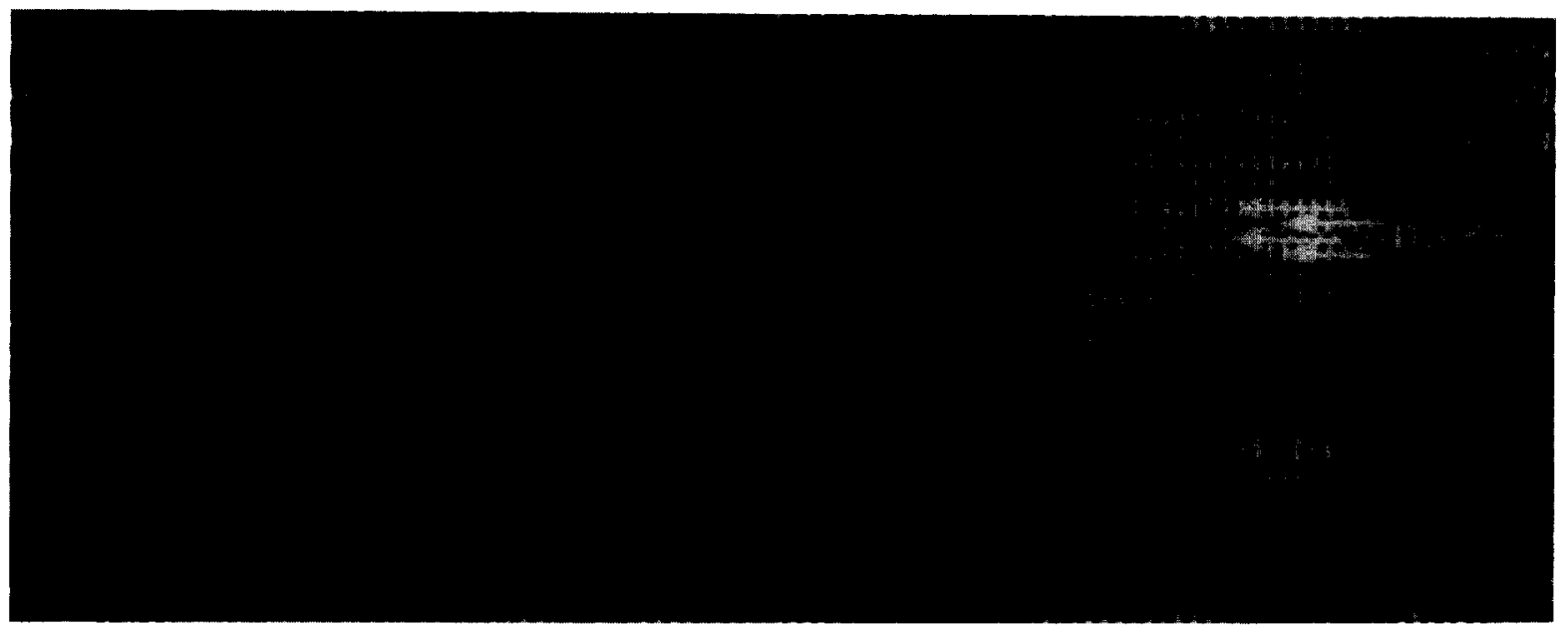

Fig. 6. HRTEM image in [0 10 ] orientation in the area with high defect density. Looking under grazing incidence along the arrows clearly reveals the local jumps and bending of the atom planes.

to be more faulted. Diffraction experiments show that in some regions in plane rotation of the $(001)$ planes with a $45^{\circ}$ angle have occurred although the $c$-axis orientation is still good. The transition temperature of this sample is only $84.8 \mathrm{~K}$. This indicates the strong influence of the deposition condition.

Closing the discussion concerning the TEM experiment, we remark that the interface between YBCO and PBCO layers could not be detected. Usually it could be realized by a slightly different contrast. In our samples the contrast is quite uniform across the interface and the defect contrast could further obscure the difference in contrast between the YBCO and PBCO layers. Well defined interfaces, however, were determined by various analysis techniques, e.g., RBS, SAM, He-ion channeling, and SIMS, as we will described in more detail in the following interdiffusion studies.

To study the diffusion occurring at the interfaces a number of samples have been analyzed. RBS experiments provide information on the interdiffusion of the $\mathrm{YBCO} / \mathrm{PBCO} / \mathrm{YBCO}$ heterostructures. An example of a $\mathrm{YBCO} / \mathrm{PBCO} / \mathrm{YBCO}$ trilayer with thickness sequence of 114,34 , and $40 \mathrm{~nm}$ is shown in fig. 3. The random spectrum was simulated using the RUMP computer code as a model of $\mathrm{ZrO}_{2}$ substrate covered by a YBCO/PBCO/YBCO multilayer. The RBS computer simulation curve of the stoichiometric layers for the given thicknesses fits well to the experimental data. This suggests that the overall film has the right stoichiometry. The onset of the backscattered ions for the different elements at the corresponding surfaces are indicated by arrows. $Y_{t}$ and $Y_{b}$ refer to the rise of the $Y$-signal at the surface of the top and the bottom YBCO layer, respectively. Within the depth resolution limits of RBS of around $5 \mathrm{~nm}$, no interdiffusion between layers can be observed.

The results of SAM are given in figs. 7 and 8 . An Auger electron spectroscopy (AES) sputter profile taken on a $\mathrm{YBCO} / \mathrm{PBCO} / \mathrm{YBCO}$ multilayer is shown in fig. 7. Carbon is seen only at the surface of the multilayer structure; inside the multilayer the $\mathrm{C}$ peak-

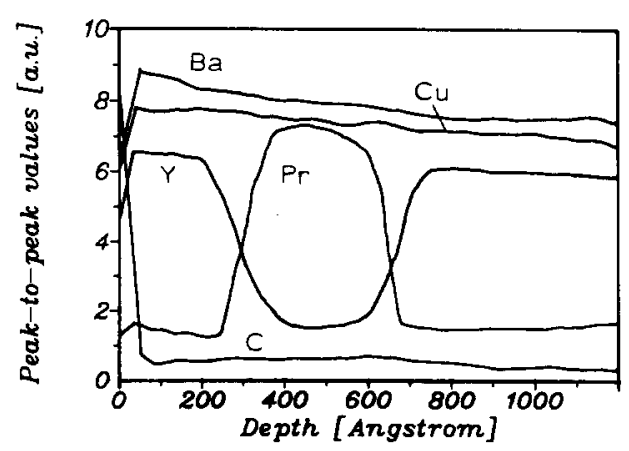

Fig. 7. The Auger sputter profile of a YBCO/PBCO/YBCO trilayer on $\mathrm{ZrO}_{2}$ substrate. The sputter rate is calibrated to be 25 $\mathrm{nm} / \mathrm{min}$. 


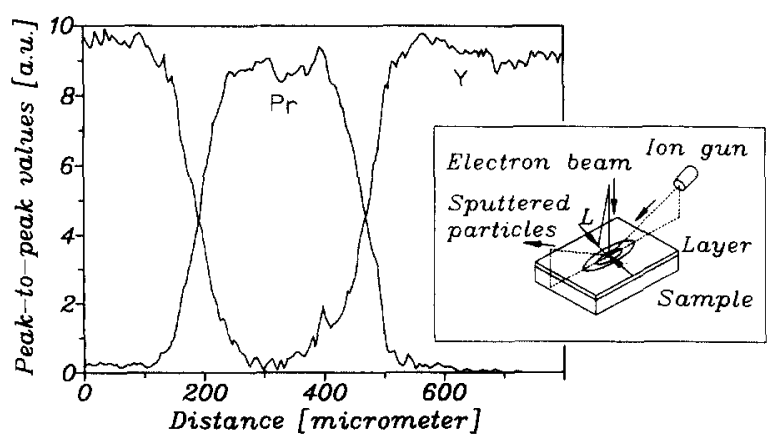

Fig. 8. Auger crater-edge line profile of a YBCO/PBCO/YBCO trilayer sample. The elements praseodymium and yttrium are shown. The inset is the schematic diagram of the crater-edge line profiling. The angle between the crater edge surface and sample surface is $\sim 0.04^{\circ}$ so the crater edge provides an about $10^{4}$ times enlarged picture of the perpendicular depth.

to-peak values reach noise level. For the elements $\mathrm{Ba}$, $\mathrm{Cu}$ and $\mathrm{O}$, constant peak-to-peak values and, therefore, constant concentrations are found throughout all three layers. In the YBCO top and bottom layer, the Pr peak-to-peak values reach noise level. Meanwhile the Y peak-to-peak values reach noise level in the PBCO layer. As a measure for the resolution $r$, the distance between 20 and $80 \%$ of the maximum peak-to-peak value can be taken (see fig. 7). Using the same instrument settings the sputter rate of the ion gun is calibrated with a $100 \mathrm{~nm} \mathrm{Ta}{ }_{2} \mathrm{O}_{5}$ layer on a Ta substrate. From this calibration the estimated depth resolution for $\operatorname{Pr}$ is $7 \mathrm{~nm}$. This result is compatible with RBS.

With Auger crater edge profiling we are able to stretch the interfaces by about a factor of $10^{4}$. Knowing the thickness of the PBCO layer the tangent of the angle between the normal to the sample surface and the normal to the crater edge surface (see the inset diagram in fig. 8 ) can be calculated and equals $\sim 0.001$. In this way very detailed information about the composition of interfaces can be obtained. In fig. 8 crater edge line profiles are given for $\mathrm{Y}$ and $\mathrm{Pr}$. As in the case of the AES sputter profile, in the YBCO top and bottom layer, the Pr peak-to-peak values reach noise level. The $Y$ peak-to-peak values reach noise level in the PBCO layer. The thicknesses on the bottom axis were calculated using the thicknesses found in the AES sputter profile (fig. 7). If we calculate the resolution $r$ for the Pr line profile we find $r=7 \mathrm{~nm}$. Thus the crater edge profiling revealed a very clear interface as well as RBS and SAM.

In addition, these results have been verified by SIMS depth profiling through a $\mathrm{YBCO} / \mathrm{PBCO} /$ YBCO trilayer. For this measurement a Cameca ims $4 \mathrm{f}$ secondary-ion mass spectrometer was used. A wellfocused $3 \mathrm{keV} \mathrm{O}_{2}^{+}$primary ion beam with a current of $0.13 \mu \mathrm{A}$, rastered over a $250 \times 250 \mu \mathrm{m}^{2}$ area, was employed. To suppress contributions of cluster ions, only secondary ions with an energy in excess of 40 $\mathrm{eV}$ are analyzed. The detection of the positive secondary ions ${ }^{89} \mathrm{Y}^{+},{ }^{137} \mathrm{Ba}^{+},{ }^{63} \mathrm{Cu}^{+}$and ${ }^{141} \mathrm{Pr}^{+}$are given in fig. 9. The resolution of $\operatorname{Pr}$ equals $8 \mathrm{~nm}$. We did not find any evidence of $\mathrm{Pr}$ in the two YBCO layers. The result is in fairly good agreement with the above measurements.

The results on interdiffusion presented here demonstrate that in the $\mathrm{YBCO} / \mathrm{PBCO} / \mathrm{YBCO}$ multilayer no pronounced interdiffusion has occurred. A combination of RBS, SAM and SIMS reveals that interfaces are very sharp and well defined. The maximum interdiffusion depth between $\mathrm{YBCO}$ and PBCO layers is estimated to be less than $7 \mathrm{~nm}$, according to the experimental resolution of RBS and SAM. Thus we can conclude that even if any interdiffusion takes place, it would be limited to a few nanometers. This is consistent with the results in refs. [14-16], where YBCO/PBCO multilayers were fabricated by pulsed laser deposition (PLD) and having individual layer thicknesses up to one unit cell $(1.16 \mathrm{~nm})$. These results imply low interdiffusion and smooth surfaces and support our results.

For most electronic applications a very smooth film surface is required. To avoid the current leakage

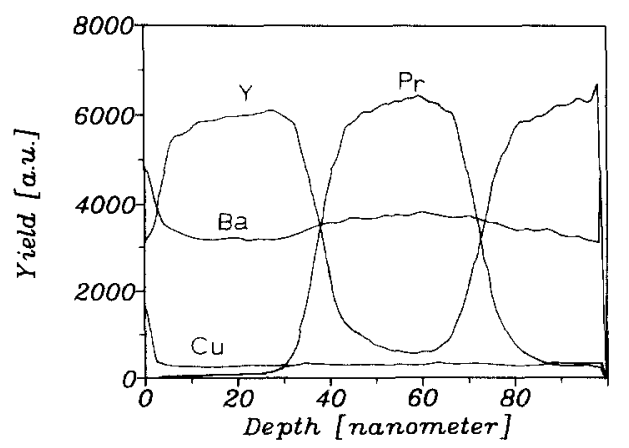

Fig. 9. Depth profile through a YBCO/PBCO/YBCO trilayer by a SIMS. Only the positive secondary ions $\mathrm{Pr}, \mathrm{Ba}, \mathrm{Cu}$ and $\mathrm{Y}$ were analyzed. 
through the barrier layer, it should be free of pinholes as well. The surface of the PBCO and YBCO top layer have been examined by SEM on $\mathrm{PBCO} /$ $\mathrm{YBCO}$ and $\mathrm{YBCO} / \mathrm{PBCO} / \mathrm{YBCO}$ multilayers samples. In fig. 10(a) the SEM photograph of a typical YBCO/PBCO/YBCO trilayers sample showed a smooth surface. The thickness of the grown films is very homogeneous over the whole substrate area $\left(6 \times 12 \mathrm{~mm}^{2}\right)$. The surface roughness can be made as small as a few nanometers as investigated using an $a$-step profiler.
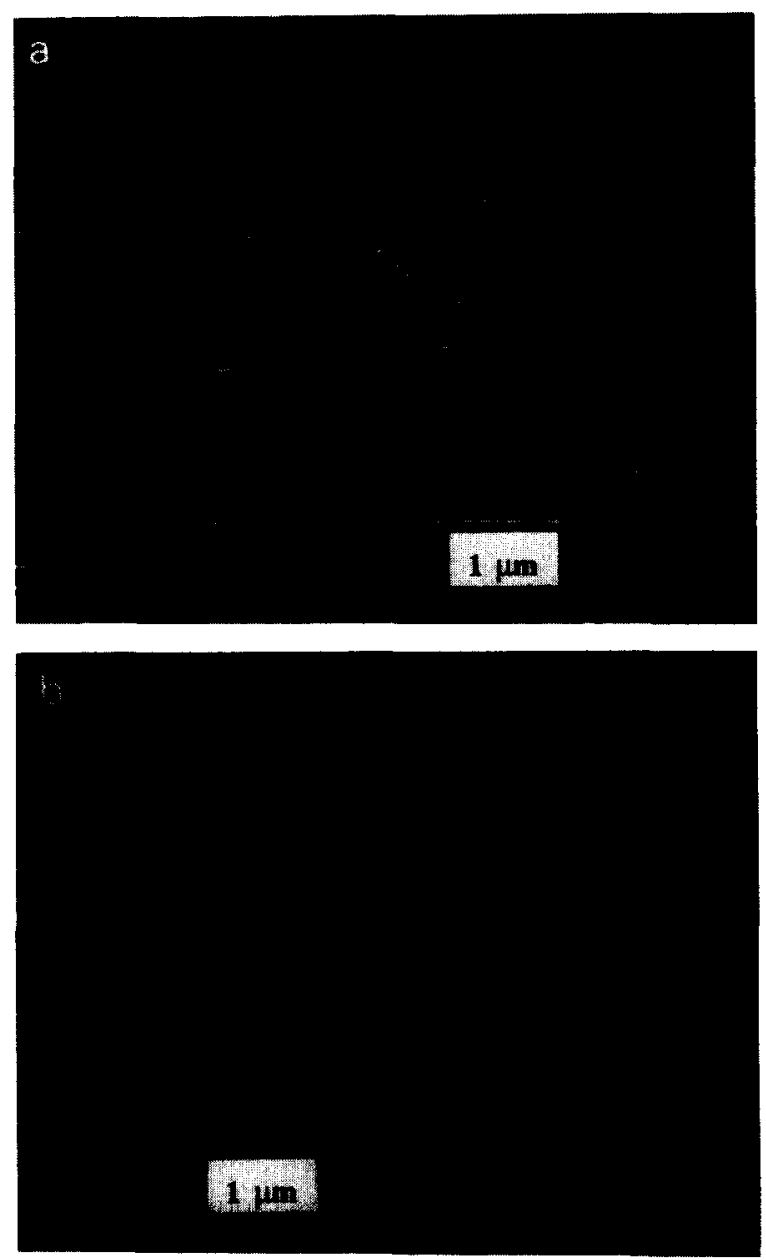

Fig. 10. (a) The SEM picture of the surface of the YBCO overlying layer of a YBCO/PBCO/YBCO trilayer sample. (b) The surface micrograph of the PBCO layer of a $\mathrm{PBCO} / \mathrm{YBCO}$ bilayer sample. The layer thicknesses are $20 \mathrm{~nm}$ PBCO and $24 \mathrm{~nm}$ YBCO, respectively.
When viewed at low magnifications, the PBCO layer also showed a smooth surface. However, closer examination reveals that the surface quality of $\mathrm{PBCO}$ layer is a little bit lower than the YBCO layer. In fig. 10 (b) we show the SEM picture of a surface of PBCO layer on a PBCO/YBCO bilayer with thicknesses of $20 \mathrm{~nm} \mathrm{PBCO}$ and $24 \mathrm{~nm}$ YBCO, respectively. Some small dots could be seen in SEM photograph. The size of these dots is around $50 \mathrm{~nm}$. It could imply that the deposition conditions for the growth of the intermediate PBCO layer is not optimum: we used the same sputter parameters as for YBCO to deposit the PBCO layer. It seems that for PBCO material the optimum deposition conditions might be different. Work is under way to minimize these small dots by varying the sputter parameters.

\section{Conclusions}

In summary, sandwich structures of $\mathrm{YBCO}$ / $\mathrm{PBCO} / \mathrm{YBCO}$ have been sputtered in situ. The epitaxy is maintained throughout the whole layer system. The orientation of the $c$-axis perpendicular to the substrate is fairly perfect. The superconductivity of the YBCO layers in these multilayer structures is not degraded compared with the individual YBCO thin films. TEM cross section photographs clearly show an excellent epitaxy between films and substrate. The film contained structural defects, which have been studied within atomic resolution. The dominant defects in the three layers appear as localized stacking faults. No signs of a pronounced lattice mismatch between YBCO and PBCO could be found. SAM, RBS, and SIMS measurements indicated that the maximum thickness of a diffusion layer between YBCO and PBCO is less than $7 \mathrm{~nm}$ according to the experimental resolution limits of RBS and SAM measurements. Thus these heterostructures provide a promising basis for superconducting electronic applications.

\section{Acknowledgements}

This work was supported by the Dutch National Research Program on High- $T_{\mathrm{c}}$ Superconductors. The SIMS experiment was done by P.C. Zalm at the Phil- 
ips Research Laboratories, Eindhoven. The interdiffusion studies in this work are part of the research program of the "Stichting voor Fundamenteel Onderzoek der Materie (FOM)", which is financially supported by the "Nederlandse organisatie voor Wetenschappelijk Onderzoek (NWO)", and were made possible by support of the "FOM-Institute voor Atoom en Molecuul Fysica (AMOLF)", Amsterdam, and the "Centrum voor Materialen Onderzoek (CMO)", Enschede.

\section{References}

[1] U. Poppe, P. Prieto, J. Schubert, H. Soltner, K. Urban and C. Buchal, Solid State Commun. 71 (1989) 569.

[2] J. Gao, W.A.M. Aarnink, G.J. Gerritsma and H. Rogalla, Physica C 171 (1990) 126.

[3] J. Gao, W.A.M. Aarnink, G.J. Gerritsma and D. Veldhuis and H. Rogalla, Proc. ASC 1990, IEEE Trans. Magn. 27 (2) (1991).

[4] J. Gao, B. Häuser and H. Rogalla, J. Appl. Phys. 67 (1990) 2512.
[5] L.R Doolittle, Nucl. Inst. and Meth. B 9 (1985) 344.

[6] Phi Multiprobe 600/595 Video tape guide, Physical Electronics Division, Perkin Elmer.

[7] J. Gao, W.A.M. Aarnink, G.J. Gerritsma and H. Rogalla, Appl. Surf. Sci. 46 (1990) 74.

[8] J. Schubert, H. Soltner, C.L. Jia, B. Kabius, B. Kabius, U. Poppe and C. Buchal, Proc. of the $\mathrm{HT}_{\mathrm{c}}$-Ustron 89.

[9] S.K. Streiffer, C.B. Eom, J.C. Bravman and T.H. Geballe, Proc. 12th Int. Congr. for Electron Microscopy (1990) Seattle, USA, vol. 4, p. 6.

[10] A.F. Marshall, R.W. Barton, K. Char, A. Kapitulnik, B. Oh and R.H. Hammond, Phys. Rev. B 37 (1988) 9353.

[11] K. Char, M. Lee, R.W. Barton, A.F. Marshall, I. Bozovic, R.H. Hammond, M.R. Beasley, T.H. Geballe and A. Kapitulnik, Phys. Rev. B 38 ( 1988 ) 834.

[12] D.E. Morris, J.H. Nickel, B. Fayn, A.G. Markelz, R. Gronsky, M. Fendorf and C.P. Burmester, Mater. Res. Soc. Symp. Proc. 169 (1990).

[13] S. Jin, T.H. Tiefel, S. Nakahara, J.E. Garebner, H.M. O'Bryan, R.A. Fastnacht and G.W. Kammlott, preprint.

[14] J.-M. Triscone, $\varnothing$. Fischer, O. Brunner, L. Antognazza, A.D.Kent and M.G. Karkut, Phys. Rev. Lett. 64 (804) 1990.

[15] Q. Li, X.X. Xi, X.D. Wu, A. Inam, S. Vadlamannati, W.L. McLean, T. Venkatesan, R. Ramesh, D.M. Hwang, J.A. Martinez and L. Nazar, Phys. Rev. Lett. 64 (3086) 1990.

[16] D.H. Lowndes, D.P. Norton and J.D. Budai, Phys. Rev. Lett. $65(1160) 1990$. 